\title{
Sur les Filaires Splendidofilariinae du genre Aproctella (1)
}

\author{
par O. BAIN *, G. PETIT *, W. J. KOZEK * * et A.-G. CHABAUD * \\ * Laboratoire de Zoologie-Vers, associé au C.N.R.S., Muséum National d'Histoire Naturelle, \\ 43, rue Cuvier, F 75231 Paris Cedex 05. \\ * International Center for Medical Research, Tulane University-Colciencias, \\ Apartado Aereo 5390, Cali, Colombia (2)
}

RESUME. Description d'Aproctella alessandroi n. sp., parasite de divers Passériformes en Colombie et en Guadeloupe, et d'une autre espèce très proche mais à microfilaire plus petite, A. golvani n. sp., parasite d'un Fringille en Guadeloupe.

Réalisation du cycle d'A. alessandroi chez Aedes togoi (développement en 13 jours dans les muscles alaires; stade I cylindrique; stade III long de 1330 à $1600 \mu \mathrm{m}$; jonction œsophage-intestin nette).

La biologie larvaire d'Aproctella, très semblable à celle de Cardiofilaria, confirme l'étroite parenté de ces 2 genres. Elle s'oppose par contre à celle de 2 autres genres de Splendidofilariinae d'Oiseaux, Splendidofilaria et Chandlerella (vecteurs : Culicoides et Simulies ; stade III long de 400 à $600 \mu \mathrm{m}$; jonction œsophage-intestin indistincte). Chez ces deux derniers genres, la biologie larvaire présente de remarquables similitudes avec celle des Lemdaninae de Reptiles et d'Oiseaux (Saurositus, Eufilaria). Il ne semble donc pas qu'il y ait de séparation nette entre les Splendidofilariinae et les Lemdaninae.

\section{On the Filariae of the genus Aproctella (Splendidofilariinae).}

SUMMARY. Aproctella alessandroi n. sp., parasitic in various Passeriformes in Colombia and Guadeloupe, and A. golvani n. sp., a similar species with smaller microfilariae parasitic in sparrows in Guadeloupe, are described. Santé.

(1) Ce travail a pu être effectué grâce à une subvention de l'Oganisation Mondiale de la

(2) Supported by Grant AI-10050 from the NIH.

Accepté le 2 septembre 1980.

Annales de Parasitologie humaine et comparée (Paris), t. 56, $\mathrm{n}^{\circ} 1$. 
The life cycle of A. alessandroi was studied in Aedes togoi (development in 13 days in the flight muscles; first-stage larva cylindrical ; third-stage larva 1330-1600 long; oesophago-intestinal junction distinct).

The larval biology of Aproctella is very similar to that of Cardiofilaria, confirming the close relationship of these two genera.

The biology differs from that of two other genera of Splendidofilariinae of birds, Splendidofilaria and Chandlerella (vectors: Culicoides and Simuliids; development in flight muscles and haemocoel; first stage larva almond-shaped; third stage larva 400-600 $\mu \mathrm{m}$ de long; oesophago-intestinal junction indistinct).

In these latter genera, the larval biology is remarkably similar to that of Lemdaninae of reptiles and birds (Saurositus, Eufilaria). It seems there is not a distinct separation between the Splendidofilariinae and the Lemdaninae.

\section{Introduction}

La systématique des Filaires est malaisée étant donnée l'atrophie des organes, et il est difficile de savoir si les caractères génériques utilisés ont une valeur phylétique ou s'ils sont de simples convergences. Cela est particulièrement vrai pour les groupes les plus évolués: Splendidofilariinae et Lemdaninae.

L'ontogénèse larvaire et la morphologie des stades infestants paraissent être les éléments d'appréciation les plus sûrs pour tenter de résoudre ces difficultés et les données dans ce domaine progressent rapidement.

La biologie larvaire du Splendidofilariinae Aproctella Cram, 1931, a été élucidée, en réalisant chez un moustique le cycle d'une nouvelle espèce, originaire de Colombie et elle semble apporter des éléments utilisables pour la classification du groupe.

\section{I - Description d'Aproctella alessandroi n. sp.}

Un Thraupis episcopus Linné, 1766, capturé à Pichinde (Etat de Valle), a deux sortes de microfilaires sanguines, différentes par la taille. A la mort de l'Oiseau, plusieurs Filaires du genre Aproctella sont récoltées dans la cavité générale ; les spécimens montrent des signes de dégénérescence (hypoderme et musculature jaunie ; utérus presque vides); une des $₹$ a quelques microfilaires dans l'ovéjecteur qui correspondent à la plus longue des microfilaires sanguines *.

* La petite microfilaire paraît correspondre à une espèce d'Eufilaria dont les adultes n'ont pas été trouvés. 
Un autre Thraupidé, Tachyphonus rufus (Bauddaert, 1783), capturé non loin de Pichinde, à Penas Blancas, a des microfilaires sanguines identiques à celles de l'Aproctella de $T$. episcopus, mais à l'autopsie de l'oiseau, aucun adulte n'est trouvé.

Le matériel comprend donc 7 Filaires de $T$. episcopus : $1 q$ holotype à microfilaires, $1 \sigma^{*}$ allotype, $1 \%$ et $4 \sigma^{*}$ paratypes (lot 99 NE, entreposé au M.N.H.N.).

\section{Description.}

La morphologie est représentée sur les figures 1 et 2 . Bouche ovale ; cavité buccale à section transversale triangulaire; capsule buccale formée par 2 segments : un segment antérieur qui porte en avant, sur les faces latérales internes, 2 dents verticales plus ou moins hautes, à apex plat ou conique ; - un segment postérieur très court, mais très fortement cuticularisé, et pas plus large que le précédent.

Chez le $\delta$, modification particulière de la cuticule sur la face ventrale, dans la région postérieure enroulée: cuticule lisse en surface, mais face interne ondulée et renforcée formant, en vue ventrale, des petites bandes transversales d'aspect ponctué * Papilles caudales disposées en 2 groupes : 1 groupe péricloacal régulièrement constitué par 5 paires de papilles, une papille impaire précloacale ; 1 groupe terminal qui comprend 2 paires de papilles, ou parfois une; les phasmides sont situées à ce niveau, mais latéralement.

Chez les $q$, vagin et ovéjecteur formant un tube simple, recourbé vers l'avant; opisthodelphie.

Microfilaires sans gaine, minces; tête légèrement amincie ; crochet céphalique très exigu; corps interne court; queue régulièrement effilée, avec courte zone terminale anucléée.

Dimensions :

- $q$ holotype : corps long de $15 \mathrm{~mm}$ et large de $240 \mu \mathrm{m}$; anneau nerveux à $180 \mu \mathrm{m}$ de l'apex; capsule buccale haute de $8 \mu \mathrm{m}$; œsophage long de $450 \mu \mathrm{m}$; vulve à $1010 \mu \mathrm{m}$ de l'apex; queue longue de $180 \mu \mathrm{m}$.

- $\delta$ allotype : corps long de $10,1 \mathrm{~mm}$, large de $195 \mu \mathrm{m}$; anneau nerveux à $160 \mathrm{~m}$ de l'apex; œsophage long de $465 \mu \mathrm{m}$; queue longue de $77 \mu \mathrm{m}$; spicules gauche et droit longs de $100 \mu \mathrm{m}$ et $82 \mu \mathrm{m}$; cuticule ventrale modifiée entre 800 et $2400 \mu \mathrm{m}$ en avant de l'extrémité caudale.

— $q$ paratype : corps long de $18 \mathrm{~mm}$, large de $260 \mu \mathrm{m}$; anneau nerveux à $200 \mu \mathrm{m}$ de l'apex; capsule buccale haute de $8 \mu \mathrm{m}$; œsophage long de $550 \mu \mathrm{m}$; vulve à $2150 \mu \mathrm{m}$ de l'apex et portion impaire de l'ovéjecteur longue de $2300 \mu \mathrm{m}$; queue longue de $225 \mu \mathrm{m}$.

Un $\sigma^{7}$ paratype : corps long de $10,8 \mathrm{~mm}$, large de $175 \mu \mathrm{m}$; anneau nerveux à $190 \mu \mathrm{m}$ de l'apex; capsule buccale haute de $8 \mu \mathrm{m}$; œsophage long de $360 \mu \mathrm{m}$; queue lon-

\footnotetext{
* Une modification analogue de la cuticule s'observe sur les faces ventrale et dorsale de la région postérieure de la $q$ paratype.
} 

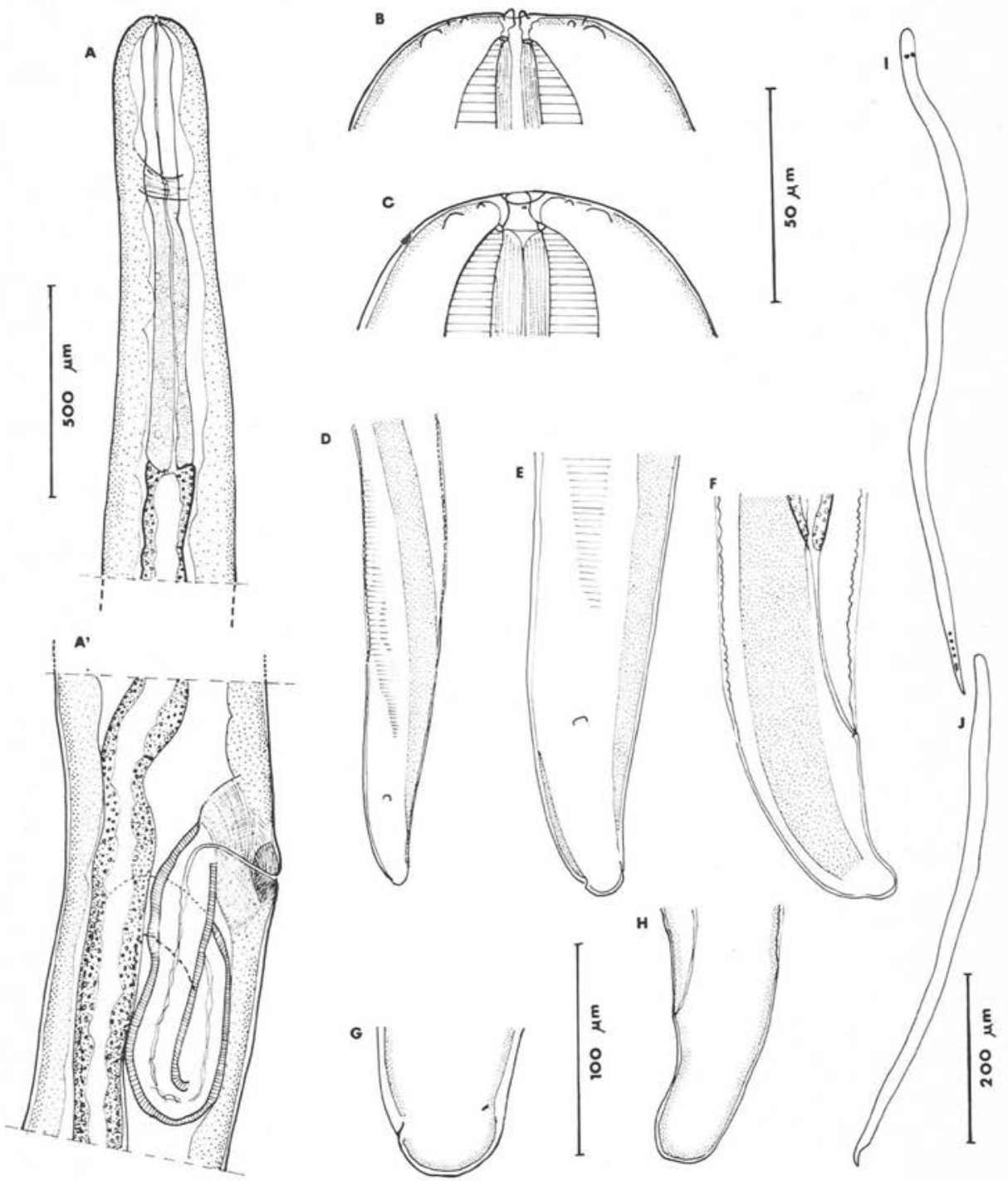

Fig. 1. A à I, Aproctella alessandroi n. sp., $q$; A et A': régions œesophagienne et vulvaire, vue latérale ; $\mathrm{B}$ et $\mathrm{C}$ : tête, vues médiane et latérale; $\mathrm{D}$ : région postérieure montrant l'ornementation cuticulaire (corde latérale ponctuée); E et $\mathrm{F}$ : région caudale, vues ventrale et latérale; $\mathrm{G}$ : extrémité caudale, vue ventrale; $\mathrm{H}$ : queue d'une autre $q$, vue latérale; $\mathrm{I}$ : microfilaire utérine ; J : Aproctella golvani $\mathrm{n}$. sp., microfilaire utérine. (D : éch. $500 \mu \mathrm{m} ; \mathrm{B}, \mathrm{C}, \mathrm{I}, \mathrm{J}$ : éch. $50 \mu \mathrm{m}$; A, A', E, F, H : éch. $200 \mu \mathrm{m}$; G : éch. $100 \mu \mathrm{m}$ ). 

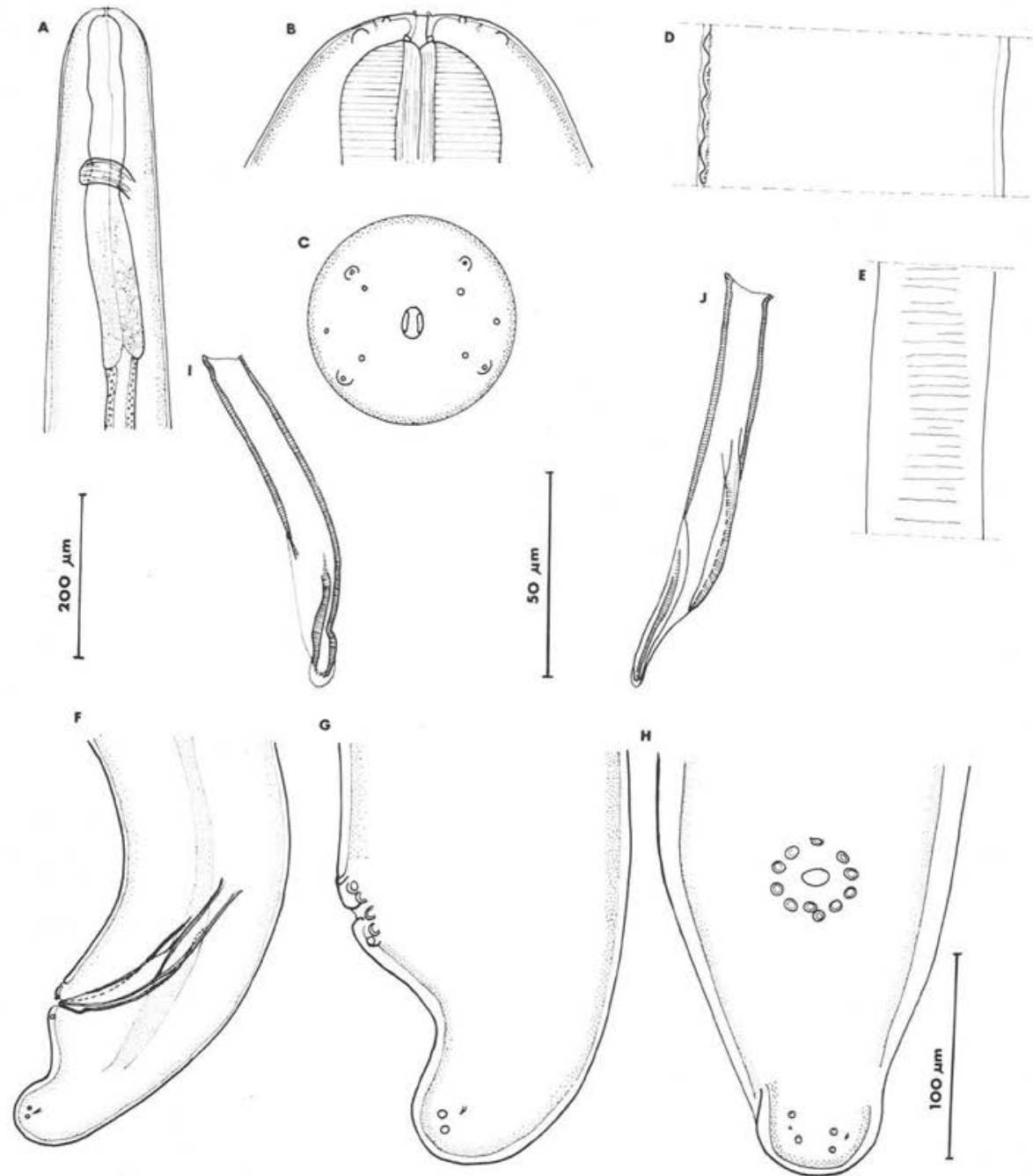

Fig. 2. Aproctella alessandroi n. sp., ơ; A : région antérieure; vue latérale; B et C: tête, vues médiane et apicale; $\mathrm{D}$ et $\mathrm{E}$ : ornementation cuticulaire ventrale de la région postérieure, vues latérale et ventrale; F, G, H : région caudale, vues latérale et ventrale; I et J : spicules droit et gauche, vue latérale, face interne.

(A, E : éch. $200 \mu \mathrm{m}$; B, C, G, H, I, J : éch. $50 \mu \mathrm{m}$; D, F : éch. $100 \mu \mathrm{m}$ ). 
gue de $70 \mu \mathrm{m}$; spicules gauche et droit longs de $88 \mu \mathrm{m}$ et $76 \mu \mathrm{m}$; cuticule ventrale modifiée entre 950 et $3280 \mu \mathrm{m}$ en avant de l'extrémité caudale.

Longueurs extrêmes des $\sigma^{*}: 9,75$ à $10,8 \mathrm{~mm}$.

- Microfilaires : - dans l'utérus : 153 à $155 \mu \mathrm{m}$ de long sur 3,5 $\mu \mathrm{m}$ de large ;

— goutte épaisse de sang cardiaque : 147, 155, 157 et $155 \mu \mathrm{m}$; - sur frottis : $140 \mu \mathrm{m}$;

- microfilaires sanguines immobilisées à la chaleur : : 186, 192, 190,190,195, 186, $185,185,195$ et $187 \mu \mathrm{m}$ de long sur 3,5 à $4 \mu \mathrm{m}$ de large ; pour une microfilaire longue de $187 \mu \mathrm{m}$ et large de $4 \mu \mathrm{m}$, anneau nerveux, pore excréteur et cellule excrétrice à $35 \mu \mathrm{m}, 60 \mu \mathrm{m}$ et $70 \mu \mathrm{m}$ de l'apex ; corps interne, cellule $\mathrm{R}_{1}$ et pore anal à 112,140 et $160 \mu \mathrm{m}$ de l'apex.

\section{Discussion}

Par la présence de 2 petites dents labiales latérales, cette Filaire Splendidofilariinae se classe dans le genre Aproctella Cram, 1931. Ce genre comprend actuellement 3 espèces, proches les unes des autres.

- A. stoddardi Cram, 1931, redécrit par Anderson, 1957, parasite de divers Passériformes et de Gallinacés en Amérique du Nord ; l'espèce se distingue de notre matériel par la pointe arrondie du spicule gauche et par la plus grande taille des microfilaires (après coloration au Giemsa, 170-217 $\mu \mathrm{m}$, au lieu de 140-157 $\mu \mathrm{m}$ chez nos spécimens).

- A. milloti Chabaud et coll., 1959, parasite d'un Ptéroclidiforme endémique de Madagascar ; cette espèce se distingue de nos spécimens par le nombre et la position des papilles caudales ( 7 paires cloacales au lieu de $5 ; 1$ paire sur la partie moyenne de la queue, qui n'existe pas chez nos spécimens ; une paire de phasmides subterminales au lieu de 2 paires de papilles plus les phasmides); il semble également que la microfilaire soit un peu plus grande ( $166 \mu \mathrm{m}$ en moyenne au lieu de $154 \mu \mathrm{m}$, dans l'utérus).

- A. carinii Pereira et Vaz, 1933, parasite de Fringillidae au Brésil (Etat de Sao Paulo). Cette espèce est extrêmement proche de nos spécimens, mais les auteurs signalent et représentent des spicules gauche et droit à extrémités pointues; seul le spicule gauche est pointu chez nos spécimens, et nous préférons donc ne pas faire l'identification à carinii.

Nous désignons donc nos spécimens sous le nom d'Aproctella alessandroi n. sp., en les dédiant au professeur A. d'Alessandro, directeur du C.I.D.E.I.M., à Cali.

\section{II - Aproctella spp. en Guadeloupe}

En juin 1973, 2 lots d'Aproctella ont été récoltés chez deux oiseaux, capturés à Petit-Bourg, en Grande-Terre. 
1. Lot $375 R L: 1$ ? et 1 \%, dans la cavité générale de Quiscalus lugubris, Swainson, 1837 (Icteridae). Le $\delta$ est long de $8,4 \mathrm{~mm}$, large de $150 \mu \mathrm{m}$; anneau nerveux à $150 \mu \mathrm{m}$ de l'apex; œsophage long de $440 \mu \mathrm{m}$; queue longue de $75 \mu \mathrm{m}$; spicules gauche et droit longs de 100 et $85 \mu \mathrm{m}$. La + est longue de $16,65 \mathrm{~mm}$, large de $325 \mu \mathrm{m}$; œsophage long de $360 \mu \mathrm{m}$; vulve à $1760 \mu \mathrm{m}$ de l'apex ; queue longue de $140 \mu \mathrm{m}$. Microfilaires utérines longues de 145,157 , et $158 \mu \mathrm{m}$.

Les dimensions et la morphologie de ces spécimens (dent, papilles caudales, spicules, aspect de la cuticule dans la région ventrale postérieure du $\delta^{*}$ ) sont analogues à celles d'A. alessandroi $\mathrm{n}$. sp. et nous les assimilons à cette espèce.

2. Lot $390 R L: 3 q$ (dont l'holotype) et $1 \delta^{\star}$ allotype, dans la cavité générale de Loxigilla noctis (Linné, 1766) (Fringillidae).

La morphologie générale et les dimensions du corps des adultes sont semblables à celles d' $A$. alessandroi, mais les microfilaires utérines sont plus petites: 115,125 , 123 et $112 \mu \mathrm{m}$ (fig. $1 \mathrm{~J}$, et fig. 3).

Il s'agit donc d'une espèce nouvelle, bien caractérisée par la petite taille des microfilaires, Aproctella golvani n. sp., que nous dédions au professeur Y. Golvan, directeur du programme Bilharziose en Guadeloupe.

Dimensions :

— $q$ holotype : corps long de $15,9 \mathrm{~mm}$, large de $250 \mu \mathrm{m}$; œsophage long de $340 \mu \mathrm{m}$; vulve à $1800 \mu \mathrm{m}$ de la tête; queue longue de $180 \mu \mathrm{m}$.

- $\delta$ allotype; corps long de $11 \mathrm{~mm}$, large de $250 \mu \mathrm{m}$; anneau nerveux à $150 \mu \mathrm{m}$ de l'apex; œsophage long de $340 \mu \mathrm{m}$; queue longue de $80 \mu \mathrm{m}$; spicules gauche et droit longs de $100 \mu \mathrm{m}$ et $80 \mu \mathrm{m}$; ornementation ventrale de la cuticule s'étendant de 480 à $2200 \mu \mathrm{m}$ de l'extrémité caudale.

\section{III - Biologie larvaire d'Aproctella alessandroi}

Les Diptères gorgés sur les deux Thraupidés sont des Culicoides nubeculosus, des Aedes aegypti et des A. togoi.

Les deux espèces de microfilaires meurent chez les Culicoides; les microfilaires d'Aproctella alessandroi montrent un début de développement chez A. aegypti et se développent jusqu'au stade infestant chez $A$. togoi, mais la bouche ou l'anus sont parfois légèrement mélanisés.

Chez A. togoi, les larves s'installent dans les muscles thoraciques; à $27^{\circ}$, les formes infestantes apparaissent le $13^{\mathrm{e}}$ jour.

Stade I de 4 jours long de $140 \mu \mathrm{m}$, large de $22 \mu \mathrm{m}$; anneau nerveux et pore excréteur à 30 et $45 \mu \mathrm{m}$ de l'apex; ébauche intestinale longue de $30 \mu \mathrm{m}$; queue longue de $35 \mu \mathrm{m}$ et pointe caudale longue de $18 \mu \mathrm{m}$. 

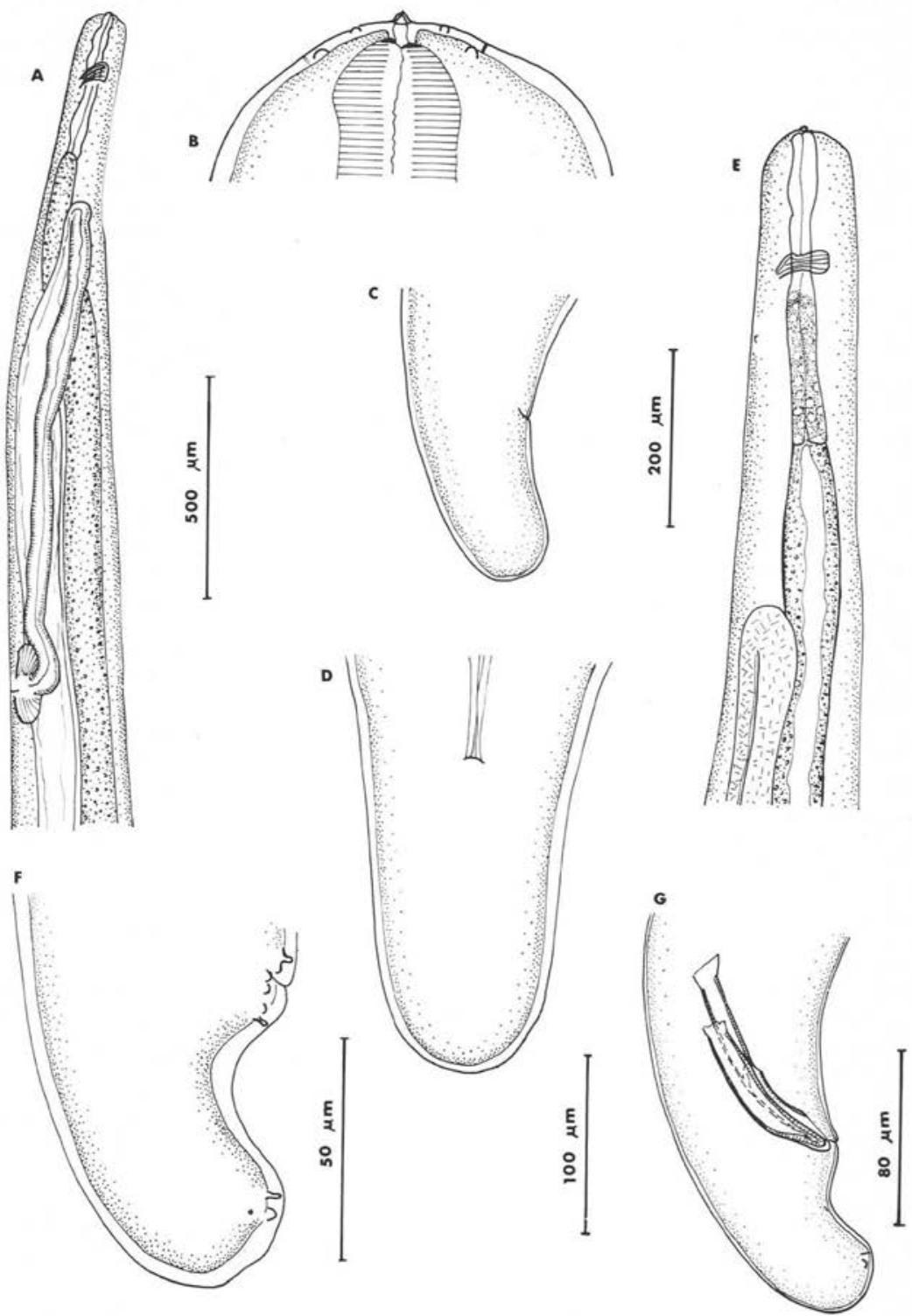

Fig. 3. Aproctella golvani n. sp.; A à D, $q$; A : région antérieure, vue latérale; $\mathrm{B}$ : tête, vue médiane; $\mathrm{C}$ et $\mathrm{D}$ : région caudale, vues latérale et ventrale; $\mathrm{E}$ à $\mathrm{G}, \delta^{\top}$ allotype ; $\mathrm{E}$ : région antérieure, vue latérale ; F : queue, vue latérale ; G : région caudale et spicules, vue latérale. (A : éch. $500 \mu \mathrm{m}$; B, D et F : éch. $50 \mu \mathrm{m}$; C et E : éch. $100 \mu \mathrm{m}$; G : éch. $80 \mu \mathrm{m}$ ). 


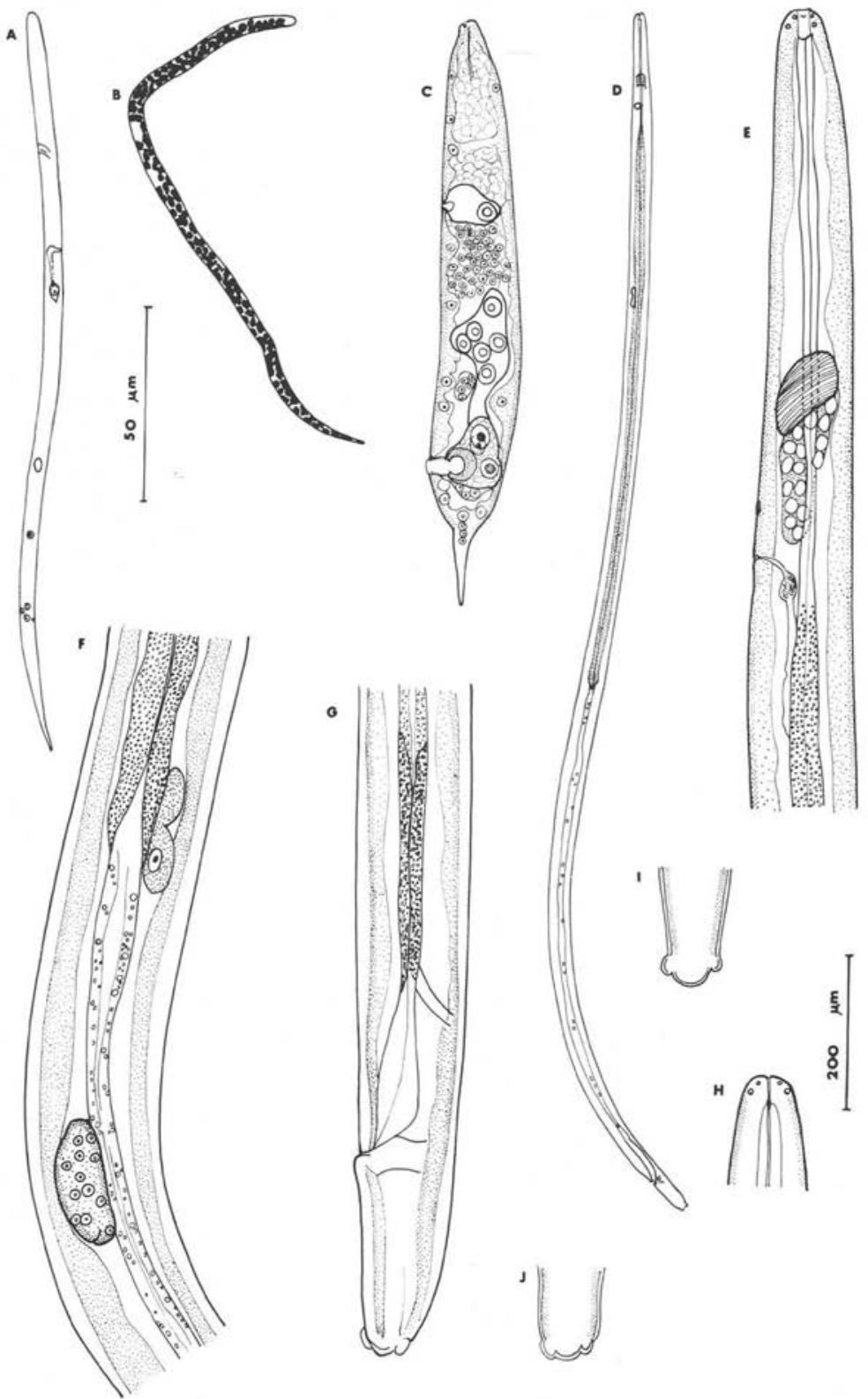

Fig. 4. Aproctella alessandroi n. sp.; A : microfilaire sanguine, coloration vitale ; B : idem, giemsa; C : stade I de 4 jours, vue latérale; D à $\mathrm{J}$ : stade infestant; D : $q$, vue latérale ; $\mathrm{E}$ : région antérieure, vue latérale; $\mathrm{F}$ : jonction œsophage-intestin, $\delta$ (formation cellulaire énigmatique contre la paroi dorsale de l'œsophage); G: région caudale, vue latérale ; $\mathrm{H}$ : tête, vue médiane; I et $\mathrm{J}$ : deux extrémités caudales, vue latérale.

(D: éch. $200 \mu$; le reste: éch. $50 \mu \mathrm{m}$ ). 
Larves infestantes*, longues de 1330 à $1600 \mu \mathrm{m}$, larges de 27 à $30 \mu \mathrm{m}$; capsule buccale haute de $7 \mu \mathrm{m}$; anneau nerveux à 105-110 $\mu \mathrm{m}$ de l'apex; œsophage divisé long de 720 à $870 \mu \mathrm{m}$, nettement distinct de l'intestin ; rectum long de 62 à $78 \mu \mathrm{m}$; queue longue de 50 à $57 \mu \mathrm{m}$, à extrémité conique-arrondie, bordée par 2 languettes latérales arrondies; ébauche génitale haute de $28 \mu \mathrm{m}$ et large de $12 \mu \mathrm{m}$, à $1400 \mu \mathrm{m}$ de l'apex chez une larve $\uparrow$ longue de $1.600 \mu \mathrm{m}$ à $850 \mu \mathrm{m}$ de l'apex chez une larve $\delta$ longue de $1720 \mu \mathrm{m}$ (fig. 4).

\section{IV - Conclusion}

Les Onchocercidae Splendidofilariinae parasites d'Oiseaux comptent 7 genres.

Pour 4 d'entre eux, le développement larvaire chez l'insecte a été réalisé par :

- Anderson (1956) pour Splendidofilaria Skrjabin, 1923.

- Dissanaike et Niles (1967) pour Cardiofilaria Strom, 1937.

- Hibler (1963) et Bartlett (1979) pour Chandlerella Yorke et Maplestone, 1926.

- Nous-mêmes pour Aproctella Cram, 1931.

Ces cycles évolutifs se répartissent en deux groupes.

Groupe A : Aproctella et Cardiofilaria.

Vecteur : Culicidae ; localisation : muscles alaires ; stade 1 : forme cylindrique ; stade infestant: long (1 200-2 $000 \mu \mathrm{m})$, épais $(28-30 \mu \mathrm{m})$, à œsophage nettement distinct de l'intestin, avec queue à extrémité conique émoussée, bordée par 2 languettes subterminales arrondies.

Groupe B : Chandlerella et Splendidofilaria.

Vecteur: Cératopogonide ou Simulie; localisation : muscle ou hémocèle; stade 1 : forme en amande ; stade infestant : court (400-600 $\mu \mathrm{m})$, gracile, avec séparation œsophage-intestin peu perceptible, à queue conique sans languettes chez Chandlerella et à queue munie de 2 appendices arrondis terminaux chez Splendidofilaria.

Le groupe $A$ est très homogène ; nous avons donc ici confirmation de la parenté des genres Aproctella et Cardiofilaria. Aproctella se distingue par la présence de dents labiales latérales et les microfilaires plus courtes (Anderson et Freeman, 1969), mais il s'agit de particularités acquises tardivement dans l'ontogénèse.

La morphologie larvaire du groupe $\mathrm{A}$ ne présente pas de caractères très particuliers.

Dans le groupe $B$, il existe de légères différences au niveau de l'extrémité caudale du stade infestant, qui pourraient avoir une valeur générique, mais l'ensemble

* La cavité pseudocoelomique des larves contient des formations plus ou moins volumineuses, situées en général vers la fin de l'œsophage et le début de l'intestin, dont la nature est inconnuc. 
reste cependant bien homogène. La morphologie larvaire, bien caractéristique, montre des affinités étroites avec celles des Lemdaninae de Reptiles et Oiseaux [Saurositus Macfie, 1924, cf. Bain (1969) ; Eufilaria Seurat, 1921, cf. Bain (1980)].

Les limites actuellement admises entre Splendidofilariinae et Lemdaninae méritent donc vraisemblablement d'être remaniées.

\section{Bibliographie}

Anderson R.C.: The life cycle and seasonal transmission of Ornithofilaria fallisensis Anderson, a parasite of domestic and wild ducks. Canad. J. Zool., 1956, 34, 485-525.

Anderson R. C.: Taxonomic studies on the genera Aproctella Cram, 1931 and Carinema Pereira and Vaz, 1933 with a proposal for a new genus Pseudaproctella n. gen. Canad. J. Zool., 1957, $35,25-33$.

Anderson R. C., Freeman R.S.: Cardiofilaria inornata (Anderson, 1956) from woodcock with a review of Cardiofilaria and related genera (Nematoda: Filarioidea). Trans. Amer. Microsc. Soc., $1969,88,68-79$.

Bain O.: Développement larvaire de Saurositus agamae hamoni n. s.-sp., Eufilariinae parasite d'Agame en Haute-Volta, chez Anopheles stephensi. Ann. Parasitol. Hum. Comp., 1969, 44, 581-594.

Bain O.: Deux Filaires du genre Eufilaria chez le Merle; développement chez Culicoides nubeculosus. Ann. Parasitol. Hum. Comp., 1980, 55, 583-590.

Bartlett C. M.: Filarioid nematodes (Filarioidea: Onchocercidae) of the crow (Corvus brachyrhynchos brachyrhynchros Brehm) in Southern Ontario. Thèse, Master of Science, Univ. Guelph, 1979, 155 p., polycopiées.

Chabaud A.-G., Anderson R.-C., Brygoo E.-R.: Sept Filaires d'Oiseaux malgaches. Ann. Parasitol. Hum. Comp., 1959, 34, 88-109.

Dissanaike A.S., Niles W. J.: On two infective filarial larvae in Mansonia crassipes with a note on other infective larvae in wild-caught mosquitoes in Ceylon. J. Helm., 1967, 41, 291-298.

Hibler C.P.: Onchocercidae (Nematoda : Filarioidea) of the american magpie, Pica pica hudsonia (Sabine), in northern Colorado. Ph. D. thesis, Colorado State University, Ft Collins, Colorado, 1963 (in Bartlett, 1979).

Pereira C., Vaz Z. : Carinema carinii, n. gen. e n. sp. de filarideo parasito do corrupiao (Xanthornus sp.) passaro fringilliformes. Rev. Biol. Hyg., 1933, 4, 56-58. 